\title{
A New Protocol Applied to Cancer Treatment- Mathematical Model-Singular Perturbed Vector Field Algorithm
}

Ophir Nave ( $\square$ naveof@gmail.com )

Jerusalem College of Technology

\section{Research Article}

Keywords: Mathematical model, Stability analysis, Reduction method, Cancer Treatment

Posted Date: February 15th, 2022

DOI: https://doi.org/10.21203/rs.3.rs-1321816/v1

License: (c) (i) This work is licensed under a Creative Commons Attribution 4.0 International License.

Read Full License 


\title{
A New Protocol Applied to Cancer Treatment-Mathematical Model-Singular Perturbed Vector Field Algorithm
}

\author{
Ophir Nave ${ }^{a}$ \\ ${ }^{a}$ Department of Mathematics, Jerusalem College of Technology
}

\begin{abstract}
In this study, we investigated the mathematical model for cancer treatment. The model takes into account the interaction of the cancer cells with the immune system and the treatment that combined unlicensed dendritic cells and anti-VEGF. We propose a new non-standard protocol for the treatment of cancer. In addition, the mathematical model includes equations of the variability of the treatments unlicensed dendritic cells and anti-VEGF as a function of the time and dependence on the Length of tumor vasculature.
\end{abstract}

Keywords: Mathematical model, Stability analysis, Reduction method, Cancer Treatment

Data: All data generated or analysed during this study are included in this published article

\section{Introduction}

This article discusses the study of cancer. Cancer - on its various issue, the number one cause of death in the Western world.

Cancer is a general name for a group of diseases consisting of dozens of different types of malignancies, characterized by a rapid and uncontrolled proliferation of cells in the body, which impairs the proper functioning of the organs in which it has spread. There are countless types of cancer that differ from each other in their manifestations, during them, in the ways of treatment, and in the chances of success. It would be a mistake to infer from one type of cancer to another. One should check and inquire about each case individually.

Corresponding author. E-mail addresses: naveof@gmail.com (O. Nave) 
The most common cancers are breast cancer in women (breast cancer also exists in men), prostate cancer (prostate), lung cancer, colon cancer, lymph node cancer (lymphoma).

The first thing is to diagnose the disease and then build a customized treatment plan for each and every patient.

The degree of malignancy and the degree of spread of the cancerous tumor must be determined in the treatment plan [1].

The degree of grading expresses the degree of violence of the tumor and is based on the morphological characteristics (shape) of the tumor when viewed under a microscope. The degree of malignancy is determined by a number of parameters, including the size of the malignant cell, the number of nuclei in the cell, the presence of nuclei within the nucleus, markers that express a rapid rate of division of the malignant cells and the degree of healthy tissue destruction by the diseased cells [2]

The degree of staging expresses the degree of tumor dispersion in the body. Is it located in one limb, or is it involving more limbs. There are several methods for estimating and scoring the degree of spread. There is not necessarily a relationship between the degree of malignancy and the degree of tumor spread. In solid malignancies in solid organs (not in the blood and lymph), it is customary to use the $T N M$ method to assess the degree of spread:

$T$ - Tumor refers to the size of the tumor.

$N$-Nodes express the extent of lymph node involvement in the vicinity of the tumor.

$M$ - Metastases Indicates the presence or absence of distant metastases.

Many researchers around the world from various fields such as Medical doctors, mathematicians, physicists, etc. try and sometimes even succeed in some cases in treating cancer. The research group is divided into several main types of research: 1: Medical Doctors and cancer researchers who run research laboratories for the study of cancer cells. Usually, these labs are in contact with drug research companies so they actually do experiments first on mice and later on humans. 2: Cancer researchers who run labs and only research the behavior of the various cancer cells. 3: Physicists, mathematicians, etc. who study cancer from the theoretical scientific aspect. That is, trying to develop mathematical models that describe the tumor and its interaction with the immune system and other cells in the body. Usually, these models also offer equations that include the way of treatment [3]-[6] . 
In general, mathematical models that describe the interaction of cancer cells with the immune system and other cells in the body are models that include a system of ordinary or partial differential equations. The equations are many and in most cases, an analytical solution cannot be obtained. Therefore in most cases, the solution is a numerical solution and various numerical simulations are applied. In some cases, these models can be studied by applying asymptotic methods such as Homotopy Analysis Method, Perturbation Method, Method of Integral Invariant Manifold, etc.

One of the important directions in cancer research is finding equilibrium points and investigating the stability of these points [7]-[10].

This is the aim of the present study. We first make a reduction to the original model without losing any biological information of the model, find equilibrium points of the reduced model, and finally investigate the stability of those points.

\section{Mathematical formulation}

In general, when developing a mathematical model related to biological, physical, chemical phenomena etc. one should take into account as many variables and parameters as possible, but on the other hand, it will be difficult to solve the model analytically but only numerically. On the other hand, if we take a few dynamical variables and parameters, the model will not reflect reality. Therefore, the current model is optimal. The mathematical model takes into account the interaction of the immune system with the cancer cells and the treatment protocol itself. The main assumptions of the model can be found at the paper [11]. The mathematical model includes nonlinear ordinary differential equations of the first order that describe the interaction between the cancer cells and the immune system. The evolution in time of the following dynamical variables are taken in the model: $T$-Number of cancer cells $([T]=$ cell $), U$-Number of mature unlicensed dendritic cells $([U]=$ cell $), D$-Number of mature licensed dendritic cells $([D]=$ cell $)$, $A_{E}$-Number of activating/proliferating effector memory $C D 8^{+} T$ cells $\left.\left(A_{E}\right]=c e l l\right), E$-Number of activated effector memory $C D 8^{+} T$ cells $([E]=$ cell $), A_{H}$-Number of activating/proliferating memory helper $C D 4^{+} T$ cells $\left(\left[A_{H}\right]=c e l l\right), H$-Number of activated memory helper $C D 4^{+} T$ cells $([H]=$ cell $), A_{R}$-Number of activating/proliferating regulatory $\mathrm{T}$ cells $\left(\left[A_{R}\right]=\right.$ cell $), R$ Number of activated regulatory $T$ cells $([R]=$ cell $), Y$ Endothelial cells number $([Y]=$ cell $)$, $C$-Concentration of $I L-2\left([C]=n g m L^{-1}\right), S$-Concentration of $T G F-\beta$ (Transforming growth factor beta) $\left([S]=n g m L^{-1}\right), I$-Concentration of $I L-10\left([I]=n g m L^{-1}\right), A_{1}$-Concentration 
of angiopoietin-1 $\left(\left[A_{1}\right]=n g m L^{-1}\right), A_{2}$-Concentration of angiopoietin-2 $\left(\left[A_{2}\right]=n g m L^{-1}\right)$, $V$-Concentration of free VEGF (Vascular endothelial growth factor) $\left([V]=n g m L^{-1}\right), V_{a^{-}}$ Concentration of anti-VEGF $\left(\left[V_{a}\right]=n g m L^{-1}\right), B$-Length of tumor vasculature $([B]=\mu m)$. The system of equations that describes the interaction between all the above parameters are as 
follows [12]:

$$
\begin{aligned}
& \frac{d T}{d t}=\gamma_{1} T\left(1-\frac{T}{\lambda_{B} B}\right)-\frac{r_{0} T}{\left(1+k_{2} T E^{-1}\right)\left(1+k_{3} R E^{-1}\right)\left(1+S S_{1}^{-1}\right)\left(1+V V_{1}^{-1}\right)} \equiv F_{1}(1) \\
& \frac{d U}{d t}=\frac{a T}{\left(\left(1+V V_{3}^{-1}\right)\left(1+I I_{1}^{-1}\right)\left(1+R R_{1}^{-1}\right)\right)}-\frac{\lambda U}{\left(1+U M_{H}^{-1}\right)}-\delta_{U} U+\mathcal{F}_{D C}(q, t) \equiv F_{2}(2) \\
& \frac{d D}{d t}=\frac{\lambda U}{1+U M_{H}^{-1}}-\delta_{D} D \equiv F_{3} \\
& \frac{d A_{E}}{d t}=\frac{\alpha_{1} M_{E}}{1+k_{4} M D^{-1}}-\delta_{A_{E}} A_{E} \equiv F_{4} \\
& \frac{d E}{d t}=\frac{\alpha_{2} A_{E} C}{\left(1+V V_{2}^{-1}\right)\left(1+S S_{2}^{-1}\right)\left(C_{1}+C\right)}-\delta_{E} E \equiv F_{5} \\
& \frac{d A_{H}}{d t}=\frac{\alpha_{3} M_{H}}{1+k_{4} M(U+D)^{-1}}-\delta_{A_{H}} A_{H} \equiv F_{6} \\
& \frac{d H}{d t}=\frac{\alpha_{4} A_{H} C}{\left(1+V V_{2}^{-1}\right)\left(1+S S_{2}^{-1}\right)\left(C_{1}+C\right)}-\frac{\alpha_{7} H S}{S_{3}+S}-\delta_{H} H \equiv F_{7} \\
& \frac{d A_{R}}{d t}=\frac{\alpha_{5} M_{R}}{1+k_{4} M D^{-1}}-\delta_{A_{R}} A_{R} \equiv F_{8} \\
& \frac{d R}{d t}=\frac{\alpha_{6} A_{R} C}{C+C_{1}}+\frac{\alpha_{7} H S}{S+S_{3}}-\delta_{R} R \equiv F_{9} \\
& \frac{d C}{d t}=\frac{p_{C} A_{H}}{\left(1+S S_{4}^{-1}\right)\left(1+I I_{2}^{-1}\right)}-\frac{C}{\tau_{C}} \equiv F_{10} \\
& \frac{d S}{d t}=p_{1} R+p_{2} T-\frac{S}{\tau_{S}} \equiv F_{11} \\
& \frac{d I}{d t}=p_{3} R+p_{4} T-\frac{I}{\tau_{I}} \equiv F_{12} \\
& \frac{d A_{1}}{d t}=\alpha_{A_{1}} B-\delta_{A_{1}} A_{1} \equiv F_{13} \\
& \frac{d A_{2}}{d t}=\frac{\alpha_{A_{2}} B T}{T+\theta_{A_{2}}}-\delta_{A_{2}} A_{2} \equiv F_{14} \\
& \frac{d V}{d t}=\alpha_{V} T+\frac{\alpha_{V_{2}} T^{2}}{T+B \theta_{V}}-\delta_{V} V-\tau V_{a} V \equiv F_{15} \\
& \frac{d Y}{d t}=\frac{\alpha_{Y} Y V}{V+Y \theta_{V_{a}}}-\frac{\omega Y A_{1} V}{\left(A_{1}+A_{2} \theta_{B}\right)(\rho Y+V)}-\delta_{Y} Y\left(1-\frac{V}{V+Y \theta_{Y}}\right) \equiv F_{16} \\
& \frac{d B}{d t}=\frac{Y \omega A_{1} V}{s\left(A_{1}+A_{2} \theta_{B}\right)(\rho Y+V)}-\frac{\gamma_{B} B A_{2}^{4} \rho Y}{\left(A_{2}^{4}+\left(A_{1} \theta_{E C}\right)^{4}\right)(\rho Y+V)} \equiv F_{17} \\
& \frac{d V_{a}}{d t}=-\left(\tau V+\delta_{V_{a}}\right) V_{a}+\mathcal{K}_{a-V E G F}(q, t) \equiv F_{18} \\
& \frac{d q_{D C}}{d t}=-\alpha_{q_{D C}} e^{q_{D C}}-\frac{\beta_{B} B}{\gamma_{q_{D C}}\left(q_{D C}^{\epsilon_{1}}-\zeta\right)}+\frac{\beta_{D C} U}{\left(B q_{D C}\right)^{\delta_{q_{1}}}-U^{\delta_{q_{2}}}} \equiv F_{19} \\
& \frac{d q_{V_{a}}}{d t}=-\alpha_{q_{V_{a}}} e^{q_{V_{a}}}-\frac{V_{a} q_{V_{a}}}{\gamma_{V_{a}}-q_{V_{a}}^{\epsilon_{2}}}+\frac{r B}{q_{V_{a}}-V_{a}^{\xi}}-\delta_{s} q_{V_{a}}^{\mu} \equiv F_{20}
\end{aligned}
$$


where the proposed personalized protocols functions are as follows

$$
\begin{aligned}
& \mathcal{F}_{D C}\left(q_{D C}(t), t\right)=q_{D C}(t) \cdot e^{\alpha_{D C} \cdot q_{D C}(t)} \sum_{k=0}^{m}\left(t-d a y_{D C} \cdot k\right)^{\gamma_{D C}} \cdot \mathcal{H}\left(t-d a y_{D C} \cdot k\right)^{-\frac{t-d a y_{D C} \cdot k}{0.5}} \\
& \mathcal{K}_{a-V E G F}\left(q_{V_{a}}(t), t\right)=q_{V_{a}}(t) \cdot e^{\alpha_{V_{a}} \cdot q_{V_{a}}}(t) \sum_{k=0}^{n}\left(t-d a y_{V_{a}} \cdot k\right)^{\gamma_{V_{a}}} \cdot \mathcal{H}\left(t-d a y_{V_{a}} \cdot k\right)^{-\frac{t-d a y_{V_{a}} \cdot k}{0.5}} .
\end{aligned}
$$

The dosages and the treatment times intervals of unlicensed dendritic cells $D C$ and the antiVEGF may vary depending on the patient, i.e., the parameters $q_{D C}, \alpha_{D C}, \operatorname{day}_{D C}, \gamma_{D C}, m$, $q_{V_{a}}, \alpha_{V_{a}}$, day $_{V_{a}}, \gamma_{V_{a}}, n$ can vary from patient to patient. The parameter day indicates the periodicity of treatment. For example if the day $=7$ then every 7 days the treatment is given to the patient.

The initial condition of the system of equations are:

$$
\begin{aligned}
& T(0)=T_{0}, U(0)=U_{0}, D(0)=D_{0}, A_{E}(0)=A_{E 0}, E(0)=E_{0}, A_{H}(0)=A_{H 0}, H(0)=H_{0}, \\
& A_{R}(0)=A_{R 0}, R(0)=R_{0}, C(0)=C_{0}, S(0)=S_{0}, I(0)=I_{0}, A_{1}(0)=A_{10}, \\
& V(0)=V_{0}, Y(0)=Y_{0}, B(0)=B_{0}, V_{a}(0)=V_{a 0}, A_{2}(0)=A_{20}, q(0)=q_{0}, Q(0)=Q_{0} .
\end{aligned}
$$

In order to facilitate the reader and prevent long writing of expressions we will briefly write the dynamic variable vector as follows: $\vec{U}=\left(T, U, D, A_{E}, E, A_{H}, H, A_{R}, R, C, S, I, A_{1}, A_{2}, V, Y, B, V_{a}, q_{D C}, q_{V_{a}}\right)$, and the right hand side of the above system we write as the vector $\vec{F}_{i}(\vec{U})$ for $i=1, \ldots, 18$. Hence the complete system of equations (1)-(20) with the initial conditions can be write as:

$$
\begin{gathered}
\frac{d \vec{U}}{d t}=\vec{F}(\vec{U}), \\
\vec{U}\left(t_{0}\right)=\vec{U}_{0} .
\end{gathered}
$$

The first step in investigating the model (1)-(20) is to reduce the number of equations without losing the biological significance of the entire model. That is, reduce the number of equations and obtain a reduced model with the same biological significance as the original model. For this purpose we apply the Global Quasi-Linearization $G Q L$ to the considered model. The second step is to find out the equilibrium points of the mathematical model and analyze their stability.

\section{Preliminary to Global Quasi-Linearization $G Q L$}

In general, problems describing natural phenomena related to biology, chemistry, physics, etc. are described by a system of multiple partial non-linear differential equations characterized by a different time change for each variable. In order to study these mathematical models, one 
must first find the hierarchy of the system. That is, to reveal the change times of each variable and thus separate the model into fast and slow subsystems. Once we reveal the hierarchy of the system, we can actually explore the fast system, while the slow system remains frozen in time as it takes time for its dynamic variables to change.

\subsection{Mathematical formulations of $G Q L$}

The Global Quasi-Linearization $G Q L$ based on the intrinsic low-dimensional manifold $I L D M$ [13]-[14]. The ILDM is a technique to simplify the study of reaction mechanisms using dynamical systems. The ILDM approach fixes a low dimensional surface which describes the slow dynamics and assumes that after a short time the fast dynamics are less important and the system can be described in lower-dimensional space.

In this section we give a brief introduction to $G Q L$ as present in the papers [15]-[16]. Given a homogeneous system of ODE:

$$
\begin{aligned}
& \frac{d \vec{V}}{d t}=\vec{G}(\vec{V}, \epsilon), \\
& \vec{V}\left(t_{0}\right)=\vec{V}_{0},
\end{aligned}
$$

where $G$ is the vector filed of the system of equations, $V \in \mathbb{R}^{\mathrm{n}}, 0<\epsilon<<1$. When $\epsilon \rightarrow 0$ then $\vec{G}(\vec{V}, \epsilon) \rightarrow \vec{G}(\vec{V}, 0)$ which belong to the fast subspace $\mathrm{W}_{\text {fast }}$, i.e. $\operatorname{Im}(\vec{G}(\vec{V}, 0)) \subset W_{\text {fast }} \subset \mathbb{R}^{\mathrm{n}}$. Let us define the following system:

$$
\begin{aligned}
& \overrightarrow{v_{f}}=A_{f} \vec{V}, \\
& \overrightarrow{v_{s}}=A_{s} \vec{V},
\end{aligned}
$$

where $u_{f}$ are the fast coordinates and $u_{s}$ are the slow one with $n_{f}$ and $n_{s}=n-n_{f}$ the dimensions respectively. And $A$ is the corresponding decomposition matrix with blocks $n \times n_{s}$ and $n \times n_{f}$ that span the fast subspace $U_{s}$ and $U_{f}$ respectively such that:

$$
\mathbb{R}^{\mathrm{n}}=\mathrm{U}_{\mathrm{f}} \oplus \mathrm{U}_{\mathrm{s}} .
$$

Using the small parameter defined at [15]:

$$
\delta=\sup _{\vec{V} \in \Omega}\left|\operatorname{Pr} r_{s} \vec{G}(\vec{V}, \epsilon)\right|
$$

where $\operatorname{Pr}$ is the projection operator. Now define the following vector field in the new coordinates $\left(v_{f}, v_{s}\right)$ :

$$
\begin{aligned}
\vec{G}_{f}\left(A_{f} \vec{V}, A_{s} \vec{V}, \epsilon\right) & =\operatorname{Pr}_{f} \vec{G}(\vec{V}, \epsilon) \\
\vec{G}_{s}\left(A_{f} \vec{V}, A_{s} \vec{V}, \epsilon\right) & =\frac{\operatorname{Pr}_{s} \vec{G}(\vec{V}, \epsilon)}{\sup _{\vec{V} \in \Omega}|\operatorname{Pr} \vec{G}(\vec{V}, \epsilon)|}
\end{aligned}
$$


According to the above definitions, the system (24) decompose to a standard Singularly Perturbed Systems (SPS) as follows:

$$
\begin{aligned}
\frac{d \overrightarrow{v_{f}}}{d t} & =\vec{G}_{f}\left(\overrightarrow{v_{f}}, \overrightarrow{v_{s}}, \epsilon\right), \\
\frac{d \overrightarrow{v_{s}}}{d t} & =\delta \cdot \vec{G}_{s}\left(\overrightarrow{v_{f}}, \overrightarrow{v_{s}}, \epsilon\right),
\end{aligned}
$$

which is the desire decomposition.

But how can one find the first decomposition practically? The following steps answer this question: - Choose $n$ vectors $u_{1}, \ldots, u_{n} \in \mathbb{R}^{\mathrm{n}}$ and are in the domain of the considered model. The vectors choose such that the vectors $\vec{G}\left(u_{1}, \epsilon\right), \ldots, \vec{G}\left(u_{n}, \epsilon\right)$ are linearly independent. Then their fast parts $\vec{G}_{f} u_{1}, \epsilon, \ldots, \vec{G}_{f} u_{n}, \epsilon$ represent a basis of $U_{f}$ for any $0<\epsilon$.

- Define the $n n$ matrix $T$ that represent quasi-linearization matrix of the vector field $\vec{G}(\vec{V}, \epsilon)$ as follows:

$$
T=\left[\vec{G}\left(u_{1}, \epsilon\right), \ldots, \vec{G}\left(u_{n}, \epsilon\right)\right]_{\text {column }} .
$$

We assume that the vector field $\vec{G}$ can be represented as a sum $\vec{G}(\vec{V}, \epsilon)=\vec{G}_{f}(\vec{V}, \epsilon)+\delta \vec{G}_{s}(\vec{V}, \epsilon)$ where $\vec{G}_{f}$ is the fast part of the vector field and $\delta \vec{G}_{s}$ is the slow part due to projectors above, then the matrix $T$ is decomposed as a sum of fast parts and slow parts as follows:

$$
T=T_{f}+\delta \cdot T_{s}=\left[\vec{G}_{f}\left(u_{1}, \epsilon\right), \ldots, \vec{G}_{f}\left(u_{n}, \epsilon\right)\right]_{\text {column }}+\delta \cdot\left[\vec{G}_{s}\left(u_{1}, \epsilon\right), \ldots, \vec{G}_{s}\left(u_{n}, \epsilon\right)\right]_{\text {column }} .
$$

Thus, asymptotically, i.e., $\delta \longrightarrow 0$, the matrix $T$ has two groups of eigenvalues: $n_{f}$ large eigenvalues that correspond to fast eigenvectors which asymptotically form a basis of the fast subspace $U_{f}$ and small $n_{s}$ eigenvalues that correspond to fast eigenvectors which asymptotically form a basis of the fast subspace $U_{s}$. The small parameter of the system that rewritten in the new coordinates using the eigenvectors can be choose as the ratio between the smallest eigenvalue in the large group and largest eigenvalue in the small group i.e., $\delta=\frac{\lambda_{f}}{\lambda_{s}}$.

\section{Analysis and Results}

In this section, we applied the $G Q L$ method to the mathematical model (1)-(20). This method enables us to transform the mathematical model to a new coordinate where the hierarchy of the new model is exposed. This procedure decomposes the mathematical model into fast and slow subsystems. We investigate the stability of the equilibrium points only to the fast subsystem while the slow subsystem remains constant. After we had found the stable equilibrium points, we inverse transformed the only the stable points and obtained the stable equilibrium points of 
the original model. In addition, we apply numerical simulation to the full model for different protocols.

\subsection{Equilibrium Points and Stability Analysis}

The GQL method decompose the mathematical model into fast and slow subsystems with an explicit hierarchy in the new coordinates, i.e., the mathematical model rewritten in a new coordinates using the eigenvectors of the transform matrix $T$ has an explicit hierarchy.

Assumed that in the new coordinates $\vec{x}=\left(x_{1}, \ldots, x_{n}\right)$ the mathematical model decomposed into the following $S P S$ form:

$$
\begin{aligned}
& \delta_{1} \cdot \frac{d x_{f}^{1}}{d t}=G_{f}^{1}\left(x_{f}^{1}, \ldots, x_{f}^{i}, x_{s}^{i+1}, \ldots, x_{s}^{n}\right) \\
& \delta_{2} \cdot \frac{d x_{f}^{2}}{d t}=G_{f}^{2}\left(x_{f}^{1}, \ldots, x_{f}^{i}, x_{s}^{i+1}, \ldots, x_{s}^{n}\right) \\
& \cdot \\
& \cdot \\
& \delta_{i} \cdot \frac{d x_{f}^{i}}{d t}=G_{f}^{i}\left(x_{f}^{1}, \ldots, x_{f}^{i}, x_{s}^{i+1}, \ldots, x_{s}^{n}\right) \\
& \frac{d x_{s}^{i+1}}{d t}=G_{s}^{i+1}\left(x_{f}^{1}, \ldots, x_{f}^{i}, x_{s}^{i+1}, \ldots, x_{s}^{n}\right) \\
& \\
& \quad \cdot \\
& \frac{d x_{s}^{f}}{d t}=G_{s}^{n}\left(x_{f}^{1}, \ldots, x_{f}^{i}, x_{s}^{i+1}, \ldots, x_{s}^{n}\right),
\end{aligned}
$$

i.e., the fastest variables are $x_{f}^{1}, \ldots, x_{f}^{i}$ and the slowest are $x_{s}^{i+1}, \ldots, x_{s}^{n}$ in the new coordinates. The decomposition to the fast and slow subsystems is due to the hierarchy of the eigenvalues of the considered matrix $T, \lambda_{1}, \lambda_{2}, \ldots, \lambda_{i}, \lambda_{i+1}, \ldots, \lambda_{n}$ where $\lambda_{i}>>\lambda_{i+1}$.

Abbreviated writing of the model (34) is:

$$
\begin{aligned}
\vec{\delta} \cdot \dot{\vec{x}}_{f} & =\vec{G}_{f}(\vec{x}) \\
\dot{\vec{x}}_{s} & =\vec{G}_{s}(\vec{x})
\end{aligned}
$$

where $\vec{x}=\left(x_{f}^{1}, \ldots, x_{f}^{i}, x_{s}^{i+1}, \ldots, x_{s}^{n}\right)$.

According to the theory of $G Q L$, the investigation of the stability analysis can be a focus on the reduced model, i.e., on the fast subsystem. As we mention above, the stability analysis can be done on the fast subsystem. For this purpose we set $\delta=0$ and get the fast manifold of the 
system. This procedure enable us to find the equilibrium points of the sub-model.

$$
\vec{G}_{f}(\vec{x})=0 \text {. }
$$

This system is an algebraic system that can be solved analytically or numerically (depending on the complexity of the system). According to the $G Q L$ method, we solve the subsystem for the fast variables while the slow variables remain constant and can be take as the initial conditions of the model rewritten in the new coordinates, i.e., we transform the initial conditions (22) using the eigenvectors of the considered matrix $T$.

\section{The algorithm for stability analysis of the equilibrium points is:}

1 : Set $\delta=0$ and obtain the fast manifold $\vec{G}_{f}(\vec{x})=0$

2: Transform the initial conditions of the considered model, and take only the slow variables: $x_{s}^{i+1}\left(x_{s 0}^{i+1}\right), \ldots, x_{s}^{n}\left(x_{s 0}^{n}\right)$

3 : Solve the algebraic system for the fast variables: $\vec{G}_{f}\left(x_{f}^{1 *}, \ldots, x_{f}^{i *}, x_{s}^{i+1}\left(x_{s 0}^{i+1}\right), \ldots, x_{s}^{n}\left(x_{s 0}^{n}\right)\right)=0$, where $^{*}$ for $j=1, \ldots, i$ denotes the equilibrium points of the fast variables and $x_{s 0}^{j}$ for $j=$ $i+1, \ldots, n$ denotes the transform of the initial conditions of the slow variables.

4: Substitute the fast equilibrium points $x_{f}^{1 *}, \ldots, x_{f}^{i *}$ from step 3 into the slow manifold $\dot{\vec{x}}_{s}=$ $\vec{G}_{s}\left(\vec{x}^{*}\right)$

5 : Solve the algebraic system $\vec{G}_{s}\left(x_{f}^{1 *}, \ldots, x_{f}^{i *}, x_{s}^{i+1 *}, \ldots, x_{s}^{n *}\right)=0$ and obtain the slow equilibrium points

6 : Compute the eigenvalues of the Jacobian matrix of the considered model at each equilibrium point from step $5: \frac{\partial\left(G_{f}\left(\vec{x}_{f}, \vec{x}_{s}\right), G_{s}\left(\vec{x}_{f}, \vec{x}_{s}\right)\right)}{\partial\left(x_{f}^{1}, \ldots, x_{f}^{i}, x_{s}^{i+1}, \ldots, x_{s}^{n}\right)} \mid\left(\vec{x}_{f}^{*}, \vec{x}_{s}^{*}\right)$

7 : The eigenvalues with negative real parts are locally stable.

8 : Inverse transform the stable points to the original coordinates using the eigenvectors of the matrix $T$

Here, in more details we present the steps for finding the fixed points in the new coordinates and determining their stability.

1. substitute the slow variables as a constant into the fast subsystem (one can take the initial condition of the slow variables as the constants).

2. setting all fast variables derivatives to zero i.e., solve the fast subsystem (with slow variables as constants) and find the equilibrium points of the fast variables $\left(x_{1}^{*}, x_{2}^{*}\right)$

$$
\begin{gathered}
G_{\text {fast }}^{1}\left(x_{1}^{*}, x_{2}^{*}, x_{3}(0), \ldots, x_{10}(0)\right)=0, \\
G_{\text {fast }}^{2}\left(x_{1}^{*}, x_{2}^{*}, x_{3}(0), \ldots, x_{10}(0)\right)=0,
\end{gathered}
$$


(here, we denoted the fast equilibrium points with star notation). Here, we have two equations with two unknown variables $x_{1}^{*}, x_{2}^{*}$.

3. substitute the equilibrium points from step $2\left(x_{1}^{*}, x_{2}^{*}\right)$ into the slow subsystem, solve the slow subsystem and find the equilibrium points of the slow variables $\left(x_{3}^{*}, \ldots, x_{10}^{*}\right)$

$$
\begin{aligned}
& G_{\text {slow }}^{3}\left(x_{1}^{*}, x_{2}^{*}, x_{3}^{*}, \ldots, x_{10}^{*}\right)=0, \\
& G_{\text {slow }}^{4}\left(x_{1}^{*}, x_{2}^{*}, x_{3}^{*}, \ldots, x_{10}^{*}\right)=0, \\
& G_{\text {slow }}^{5}\left(x_{1}^{*}, x_{2}^{*}, x_{3}^{*}, \ldots, x_{10}^{*}\right)=0, \\
& G_{\text {slow }}^{6}\left(x_{1}^{*}, x_{2}^{*}, x_{3}^{*}, \ldots, x_{10}^{*}\right)=0, \\
& G_{\text {slow }}^{7}\left(x_{1}^{*}, x_{2}^{*}, x_{3}^{*}, \ldots, x_{10}^{*}\right)=0, \\
& G_{\text {slow }}^{8}\left(x_{1}^{*}, x_{2}^{*}, x_{3}^{*}, \ldots, x_{10}^{*}\right)=0, \\
& G_{\text {slow }}^{9}\left(x_{1}^{*}, x_{2}^{*}, x_{3}^{*}, \ldots, x_{10}^{*}\right)=0, \\
& G_{\text {slow }}^{10}\left(x_{1}^{*}, x_{2}^{*}, x_{3}^{*}, \ldots, x_{10}^{*}\right)=0 .
\end{aligned}
$$

Here, we have eight equations with eight unknown variables $x_{3}^{*}, \ldots, x_{10}^{*}$.

4. substitute the equilibrium points from steps 2 and 3 at the Jacobian matrix of the full system (the model at the new coordinates).

5. compute the eigenvalues of the Jacobian matrix of the system (the model at the new coordinates) for each set of equilibrium point (the stable points are those with a negative real part of the eigenvalues).

6. transform only the equilibrium points that are stable from steps 2 and 3 to the original coordinates using the inverse matrix of the eigenvectors, i.e., compute

$$
\vec{V}_{\text {stable }}^{*}=\mathcal{A}^{-1} \vec{U}_{\text {stable }}^{i *}
$$

where $i$ indicates for different stable equilibrium points.

It should be noted here that the equilibrium points we have received in the new coordinate system are functions of the parameters of the original model and a function of the initial conditions of the new model, i.e.,

$$
x_{i}^{*}=x_{i}^{*}\left(\mu_{A}, \mu_{A_{1}}, \ldots, g_{L} ; x_{1}(0), \ldots, x_{10}(0)\right), i=1, \ldots, 10 .
$$

Comment 1: the initial condition as well as the equilibrium points $\left\{x_{1}^{*}, x_{2}^{*}, x_{3}^{*}, \ldots, x_{10}^{*}\right\}$ are biologically meaningless as there is no biological significance to the variables in the new coordinates. Hence, in order to understand the biological meaning of the above results, we should transform these points to the original coordinates using the inverse matrix of the eigenvectors $\mathcal{A}^{-1}$. 2: Since the eigenvalues are invariant under change of coordinates, hence the stability 
equilibrium points will be remain stable equilibrium points at the original coordinates.

Stability analysis of equations (37) and (38) results in having only one equilibrium point (present as vector coordinates). The equilibrium point in the original coordinates is:

$$
\begin{aligned}
& \tilde{T}^{*}=0.0632 \cdot \gamma_{1} T_{0}-27.766 \cdot \lambda_{B} r_{0} B+0.0022 \cdot k_{2} k_{3} R_{0}-18.791 \cdot S_{1} E_{0}-0.8742 \cdot V_{0} \\
& \tilde{U}^{*}=-1.6332 \cdot a T_{0}-3.5542 \cdot V_{3} V_{0}-11.112 \cdot I_{1} I_{0}+0.0021 \cdot R_{1} R_{0}+99.676 \cdot M_{H} U_{0}-9.0933 \cdot \mathcal{F}_{D C}^{0}\left(q_{0}, t_{0}\right) \\
& \tilde{D}^{*}=1.1121 \cdot\left(\lambda+M_{H}\right) U_{0}-0.0243 \cdot \delta_{D} D_{0} \\
& \tilde{A}_{E}^{*}=0.0654 \cdot \alpha_{1} M_{E} M_{0}-0.0988 \cdot D_{0} 3.4423-1.0022 \cdot \delta_{A_{E}} A_{E 0} \\
& \tilde{E}^{*}=-102.4534 \cdot \alpha_{2} A_{E 0}-0.7667 \cdot V_{2} V_{0}+97.612 \cdot S_{2} S_{0}+89.7658 \cdot \delta_{E} E_{0} \\
& \tilde{A}_{H}^{*}=1023.3422 \cdot \alpha_{3} M_{H}+187.7832 \cdot k_{4}\left(U_{0}+D_{0}\right)-12.4423 \cdot \delta_{A_{H}} A_{H 0} \\
& \tilde{H}^{*}=-0.0883 \cdot \alpha_{4} A_{H 0} C_{0}-883.8722 \cdot \alpha_{7} H_{0} S_{0}-934.0322 \cdot \delta_{H} H_{0} \\
& \tilde{A}_{R}^{*}=2378.9393 \cdot \alpha_{5} M_{R} k_{4} M_{0} D_{0}-0.0878 \cdot \delta_{A_{R}} A_{R 0} \\
& \tilde{R}^{*}=-9.9932 \cdot \alpha_{6} C_{1} A_{R 0}-7372.838 \cdot \alpha_{7} S_{3} H_{0} S_{0}+5323.3444 \cdot \delta_{R} R_{0} \\
& \tilde{C}^{*}=926.9933 \cdot p_{C} A_{H 0} \\
& \tilde{S}^{*}=8348.0044 \cdot p_{1} R_{0}+0.3883 \cdot p_{2} T_{0} \\
& \tilde{I}^{*}=-0.0032 \cdot p_{4} R_{0}-0.0032 \cdot \tau I_{0} \\
& \tilde{A}_{1}^{*}=99883.0331 \cdot \delta_{A_{1}} A_{10} \\
& \tilde{A}_{2}^{*}=93.0032 \cdot \alpha_{A_{2}} B_{0}+93.0022 \cdot \theta_{A_{2}}-92211.0001 \cdot \delta_{A_{2}} A_{20} \\
& \tilde{V}^{*}=9322.3332 \theta_{V} B_{0}-1882.9993 \delta_{V} V_{0}+2933.4448 \cdot \tau V_{a 0} V_{0} \\
& \tilde{Y}^{*}=-0.0098 \cdot \alpha_{Y}\left(Y_{0}+V_{0}\right)-0.0044 \cdot \theta_{V_{a}}-0.0098 \cdot \omega Y_{0}+0.0034 \cdot \theta_{B}\left(A_{10}+A_{20}\right)+0.0045 \delta_{Y} Y_{0}-0.0034 \theta_{Y} \\
& \tilde{B}^{*}=-0.0001 \cdot \omega Y_{0}-0.0098 \cdot A_{1} V_{0}-0.0033 \cdot\left(A_{10}-A_{20}\right) \theta_{B}-98.0099 \cdot \gamma_{B}\left(B_{0}-A_{20}\right)+0.9883 \cdot \theta_{E C} \rho\left(Y_{0}+V_{0}\right) \\
& \tilde{V}_{a}^{*}=-9883.2223 \tau V_{0}+\delta_{V_{a}} V_{a 0}+\mathcal{K}_{a-V E G F}^{0}\left(q_{0}, t_{0}\right) \\
& \tilde{q}_{D C}^{*}=-98849.9347 \cdot \alpha_{q_{D C}} q_{D C} 0-0.9922 \cdot \beta_{B} B_{0} \gamma_{q_{D C}}\left(q_{D C} 0-\zeta\right)+9.0991 \cdot \beta_{D C} U_{0} \\
& \tilde{q}_{V_{a}}^{*}=-99323.0032 \alpha_{q_{V_{a}}} q_{V_{a} 0}-0.0021 \cdot \gamma_{V_{a}} V_{a 0}+0.0034 \cdot r B_{0} V_{a 0}-1.0003 \cdot \delta_{s} q_{V_{a}} 0
\end{aligned}
$$

where the index 0 indicates the initial conditions of the model at the original coordinates. As we see the stable equilibrium point depends on the intial conditions of the mathematical mode and parameters of the model. Therefore once all these parameters are set we can get the stable equilibrium point personalized for each and every patient. 


\subsection{Numerical Simulation, Personalized Protocols}

In this section we applied numerical simulation to the mathematical model (1)-(20) with the parameters present at tables bellow for different protocols, i.e., for different treatment protocols that are expressed at the functions (21) that take into account the dose and customized treatment times for the patient.

Figures $1-13$ present the solution profiles of the cancer cells vs. time over 3 years. Every figure present the results of applying numerical simulation for personalized treatment based on the functions (21) and experimental result of experimental results based on hospital data. The analysis based on 13 patients with cancer. As we can see from these figures all the graphs results are behave with fluctuation, because the number of cancer cells is unstable for a cancer patient and when treatment is added to this whole story then the number of cancer cells loses their equilibrium and behaves in the form of fluctuations. Also as can be seen from all the graphs, hand is a relatively good match between the numerical results and the clinical results. With a deviation of no more than a few percent. At the end of each treatment cycle, PET-CT must be performed in order to decide on how to proceed the treatment, i.e., whether to increase or decrease the dose of the drug or (and) the radiation and also to determine the new treatment cycles i.e. the time periods between treatments.

Figure 1 present the cancer cells of the first patient. This patient received the following treatment: one cycle of treatment that includes treatment once every two weeks for a month. After mechanizing a break of a month then the treatment is repeated. This patient received 3 cycles of treatment with a fixed (relatively small) dose. As can be seen from the graph at the beginning of treatment, ie in the first half year the treatment is ineffective, and this is seen by the growth of the cancer cells. After the first three cycles, the dose is significantly increased but still remains once every two weeks. The second treatment lasts about 10 months. After stopping the treatment we see that there is a growth of the cancer cells but eventually there is a significant decrease of the cancer cells to almost 0 .

Figure 2 this graph describes the aggressive treatment of a patient where the dose is high from the beginning of treatment and the treatment is given for 15 months and the time between treatments is two weeks. The treatment of this patient combined with radiation. As can be seen from the graph, the cancer cells continue to decline throughout the treatment and even after the treatment.

Figure 3 this patient received fix treatment for 15 months. Treatment is given in the hospital once a month with a fixed dose. And as can be seen from the graph the cancer cells do not change significantly and remain in the same range of number of cancer cells. After that the 
dose of the treatment was increased and the treatment was done twice a month for 4 months combined with radiation. And it can be seen from the graph that as the dose and time were increased so the cancer cells started to drop significantly.

Figure 4 this patient received treatment as patient number 3 . The graphs are seen to be identical in terms of their behavior but are different in terms of fluctuations. This patient had no fluctuating changes in the number of cancer cells. We have no biological explanation for this phenomenon other than that it is a different patient with a different background and of course with different data.

Figure 5 Treatment of this patient initially lasts 8 months. Medication only. Regular dose of medicine once every two weeks. Then added radiation therapy, the treatment lasted another 3 months and was discontinued. After treatment there was a slight increase in the cancer cells. Medication is given only for two months. The cells dropped to almost zero.

Figure $6-7$ these patients received treatment as patient number 2. The graphs are seen to be identical in terms of their behavior but are different in terms of fluctuations. This patient had no fluctuating changes in the number of cancer cells. We have no biological explanation for this phenomenon other than that it is a different patient with a different background and of course with different data. Patient number 7 received radiation therapy of varying intensity during treatment. We do not know the intensity of the radiation but it would vary as a function of the number of cancer cells.

Figure 8 The behavior of the cancer cells in this patient is similar to patients $2,6,7$. This patient also received aggressive treatment with radiation. But unlike these patients, the dose varied from one treatment cycle to another. The first cycle included two months of treatment with one drug every two weeks. Thereafter the dose was increased and treatment times remained once every two weeks. It lasted again for two months. Then the dose was reduced to the initial amount with the same periods of treatment and so it continued. That is, a treatment cycle lasts two months with varying doses from one treatment cycle to another. The doses are increased and decreased intermittently. The total treatment lasted 17 months.

Figure 9 This patient was treated for a four-month cycle drug therapy without radiation. There was then a 3-month break in treatments due to the patientâs personal constraints. The patient then returned to drug and radiation therapy. And as can be easily seen from the graph the cancer cells go down quickly to almost zero. The total treatment lasted about 10 months. After that, the patient refused to continue treatment due to the appearance of other critical diseases. The patient signed a waiver form for further treatment. 
Figure 10 this patient received the same treatment as patient number 9 .

Figure 11 this patient was diagnosed with a cancerous growth. The patient refused to receive treatment on the grounds that his body could cope with the disease on its own. After his health deteriorated, the patient agreed to receive the treatment offered to him. This happened after 14.5 months from the date of diagnosis. The patient received aggressive drug and radiation therapy. And as can be easily seen from the graph, the cancer cells are on a downward trend starting from the 450th day. It can be seen that even along the way there are some declines in cancer cells. The explanation for this is the war of the immune system in the cancer cells.

Figure 12 this patient received particularly aggressive treatment. Medication and radiation therapy for 6 months. The doses were fixed and high and were given once every two weeks. The patient then asked to lower the dose due to the severe side effects he was experiencing. Treatment was stopped after 6 months. Except for pills he received once a week from the hospital. After stopping treatment for 6.5 months, the patient returned to the treatment bed for further treatment lasting 3 months. And as you can see the cancer cells have gone down to almost zero All of the above is easy to see from the solution graph.

Figure 13 this patient did not receive treatment at all. In terms of the mathematical model this means that the two functions at equations (21) are equal to zero. The patient refused to accept the treatment offered to him. The attitude of this patient is that his immune system will treat alone with the cancerous growth. This patient has no background diseases. Healthy all his life. Without chronic or serious diseases. In graphs 14-23 we present the solutions of graphs 1-13 as a density heat map smoothly i.e., without fluctuations. The redder the area it indicates more cancer cells and the blueer the area it indicates fewer cancer cells i.e. declining. As we can see, there is a complete match between graphs 1-13 and graphs 14-23 respectively. For example, the heat map of graph 22 corresponds to the solution shown in graph 9 . At the beginning of the treatment, there are many cancer cells (graph 9), so the area in the heat map is redder. With the treatment, the cells decreases, so the heat map becomes bluer, and then there is an increase in the cancer cells and so the area in the heat map becomes redder.

All the graphs of the heat maps did not take into account the fluctuations of the solutions shown in graphs 1-13 except the heat map number 22 where the fluctuations were greater than what we defined and we got the image that corresponds to graph number 12 . 


\section{Conclusions}

In this study, we investigated the mathematical model for cancer treatment. The mathematical model takes into account the interaction of the cancer cells with the immune system and the treatment that combined unlicensed dendritic cells and anti-VEGF. We developed two analytical functions that take into account the personalized treatment such that the dosages and the time interval are controlled by the doctor. We insert these two functions into the mathematical model.

We applied the GQL algorithm to the mathematical model. This method enables us to split the model into fast and slow subsystems due to the eigenvalues of the considered vector fields of the model. After that we split the model, we investigate only the fast subsystem. We have found the equilibrium points and their stability of the reduced model. We inverse transform the stability equilibrium points to their original coordinates using the inverse matrix that contains the eigenvectors of the considered system.

In addition, we applied numerical simulation to the mathematical model and compared our results with clinical data. We present the solution profiles of the cancer cells. At the numerical simulation, we take into account different treatment that fits the clinical data. This means that we fit the mathematical model for each patient in a different manner, i.e., personalized medicine. We have found that our predictions for the personal treatment agree with the clinical data. The biggest deviation from the numerical simulation to the clinical data, in the worst case, is not over 13 percent. 


\begin{tabular}{|c|c|c|}
\hline Parameter & Value & Units \\
\hline $\mathrm{a}$ & $10^{-5}$ & day $^{-1}$ \\
\hline $\mathrm{C}_{1}$ & 0.3 & $\mathrm{ng} \mathrm{mL}^{-1}$ \\
\hline $\mathrm{I}_{1}$ & 0.4 & $\mathrm{ng} \mathrm{mL}^{-1}$ \\
\hline $\mathrm{I}_{2}$ & 0.75 & $\mathrm{ng} \mathrm{mL}^{-1}$ \\
\hline $\mathrm{k}_{2}$ & 1.2 & Unitless \\
\hline $\mathrm{k}_{3}$ & 11 & Unitless \\
\hline $\mathrm{k}_{4}$ & 0.33 & Unitless \\
\hline M & $10^{7}$ & Cell \\
\hline $\mathrm{M}_{E}$ & $3 \cdot 10^{6}$ & Cell \\
\hline $\mathrm{M}_{H}$ & $6 \cdot 10^{6}$ & Cell \\
\hline $\mathrm{M}_{R}$ & $10^{6}$ & Cell \\
\hline $\mathrm{p}_{1}$ & $1.8 \cdot 10^{-8}$ & $\mathrm{ng} \mathrm{mL}^{-1} d a y^{-1}$ cell $^{-1}$ \\
\hline $\mathrm{p}_{2}$ & $1.1 \cdot 10^{-7}$ & $\mathrm{ng} \mathrm{mL} L^{-1}$ day $^{-1}$ cell $^{-1}$ \\
\hline $\mathrm{p}_{3}$ & $1.4 \cdot 10^{-8}$ & $\mathrm{ng} \mathrm{mL}^{-1} d a y^{-1}$ cell $^{-1}$ \\
\hline $\mathrm{p}_{4}$ & $1.3 \cdot 10^{-10}$ & $\mathrm{ng} \mathrm{mL}^{-1} d a y^{-1}$ cell $^{-1}$ \\
\hline $\mathrm{p}_{C}$ & $11.7 \cdot 10^{-5}$ & $\mathrm{ng} \mathrm{mL}^{-1} d a y^{-1}$ cell $^{-1}$ \\
\hline $\mathrm{r}_{0}$ & 0.9 & day $^{-1}$ \\
\hline $\mathrm{R}_{1}$ & $2 \cdot 10^{7}$ & Cell \\
\hline s & 0.17 & $\mathrm{EC}(\mu m)^{-1}$ \\
\hline $\mathrm{S}_{1}$ & 3.5 & $\mathrm{ng} \mathrm{mL}^{-1}$ \\
\hline $\mathrm{S}_{2}$ & 2.9 & $\mathrm{ng} \mathrm{mL}^{-1}$ \\
\hline $\mathrm{S}_{3}$ & 1.7 & $\mathrm{ng} \mathrm{mL}^{-1}$ \\
\hline $\mathrm{S}_{4}$ & 0.9 & $\mathrm{ng} \mathrm{mL}^{-1}$ \\
\hline $\mathrm{V}_{1}$ & 3.5 & $\mathrm{ng} \mathrm{mL}^{-1}$ \\
\hline $\mathrm{V}_{2}$ & 2.9 & $\mathrm{ng} \mathrm{mL}^{-1}$ \\
\hline $\mathrm{V}_{3}$ & 0.14 & $\mathrm{ng} \mathrm{mL}^{-1}$ \\
\hline$\alpha_{1}$ & 23 & day $^{-1}$ \\
\hline$\alpha_{2}$ & 16 & day $^{-1}$ \\
\hline$\alpha_{3}$ & 9.9 & day $^{-1}$ \\
\hline$\alpha_{4}$ & 1.9 & day $^{-1}$ \\
\hline$\alpha_{5}$ & 5.1 & day $^{-1}$ \\
\hline$\alpha_{6}$ & 2.1 & day $^{-1}$ \\
\hline$\alpha_{7}$ & 0.022 & day $^{-1}$ \\
\hline$\alpha_{A_{1}}$ & 0.24 & $\operatorname{ng}(\mu m)^{-1} m L^{-1} d a y^{-1}$ \\
\hline
\end{tabular}




\begin{tabular}{|c|c|c|}
\hline Parameter & Value & Units \\
\hline$\alpha_{A_{2}}$ & 1.92 & $\operatorname{ng}(\mu m)^{-1} m L^{-1} d a y^{-1}$ \\
\hline$\alpha_{V}$ & $3 \cdot 10^{-6}$ & $\operatorname{ng}(\mu m)^{-1}$ cell $^{-1} d a y^{-1}$ \\
\hline$\alpha_{V_{2}}$ & $3.7 \cdot 10^{-2}$ & $\operatorname{ng}(\mu m)^{-1}$ cell $^{-1} d a y^{-1}$ \\
\hline$\alpha_{Y}$ & 0.198 & day $^{-1}$ \\
\hline$\gamma_{1}$ & 0.69 & day $^{-1}$ \\
\hline$\gamma_{B}$ & 0.8 & day $^{-1}$ \\
\hline$\theta_{A_{2}}$ & $10^{6}$ & Cell \\
\hline$\theta_{B}$ & 1 & Unitless \\
\hline$\theta_{E C}$ & 4 & Unitless \\
\hline$\theta_{V_{a}}$ & $5.9 \cdot 10^{-5}$ & $\mathrm{ngmL}^{-1}(E C)^{-1}$ \\
\hline$\theta_{V}$ & 7.5 & Cancer cell $(\mu m)^{-1}$ \\
\hline$\theta_{Y}$ & $6.8 \cdot 10^{-6}$ & $\operatorname{ngmL}^{-1}(E C)^{-1}$ \\
\hline$\lambda$ & 0.5 & day $^{-1}$ \\
\hline$\lambda_{B}$ & 7.5 & Cancer cell $(\mu m)^{-1}$ \\
\hline$\delta_{A_{E}}$ & 0.2 & day $^{-1}$ \\
\hline$\delta_{A_{H}}$ & 0.2 & day $^{-1}$ \\
\hline$\delta_{A_{R}}$ & 0.2 & day $^{-1}$ \\
\hline$\delta_{A_{1}}$ & 0.072 & day $^{-1}$ \\
\hline$\delta_{A_{2}}$ & 0.072 & day $^{-1}$ \\
\hline$\delta_{D}$ & 0.5 & day $^{-1}$ \\
\hline$\delta_{E}$ & 1 & day $^{-1}$ \\
\hline$\delta_{H}$ & 0.1 & day $^{-1}$ \\
\hline$\delta_{R}$ & 0.1 & day $^{-1}$ \\
\hline$\delta_{U}$ & 0.14 & day $^{-1}$ \\
\hline$\delta_{Y}$ & 0.198 & $\mathrm{day}^{-1}$ \\
\hline$\delta_{V_{a}}$ & 0.14 & day $^{-1}$ \\
\hline$\tau$ & $5 \cdot 10^{-8}$ & mLday $^{-1}$ \\
\hline$\tau_{I}$ & 0.05 & day \\
\hline$\tau_{S}$ & 0.07 & day \\
\hline$\tau_{C}$ & 0.08 & day \\
\hline$\omega$ & 0.576 & day $^{-1}$ \\
\hline$\rho$ & 0.0033 & $\mathrm{ngmL}^{-1}(E C)^{-1}$ \\
\hline
\end{tabular}




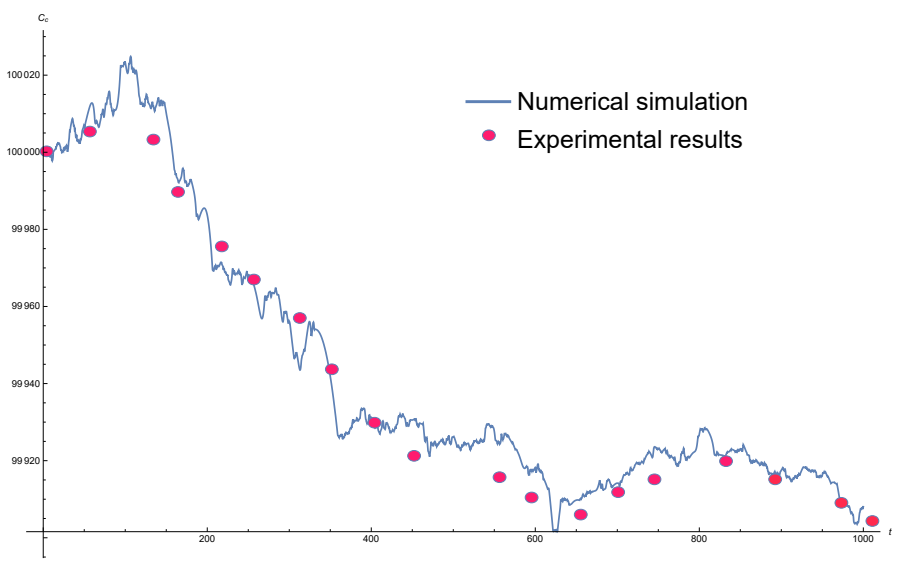

Figure 1: The solution profile of the cancer cells vs time. Patient No. 1.

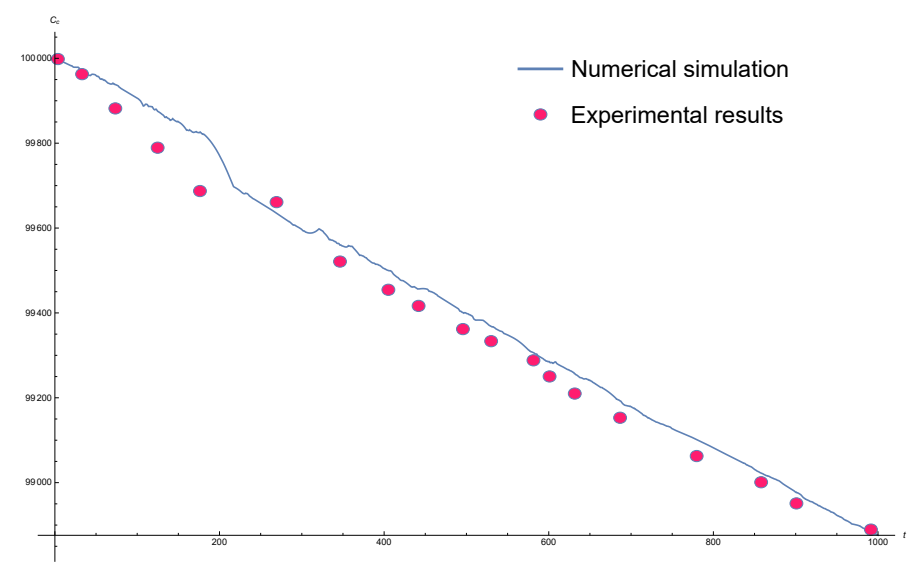

Figure 2: The solution profile of the cancer cells vs time. Patient No. 2. 


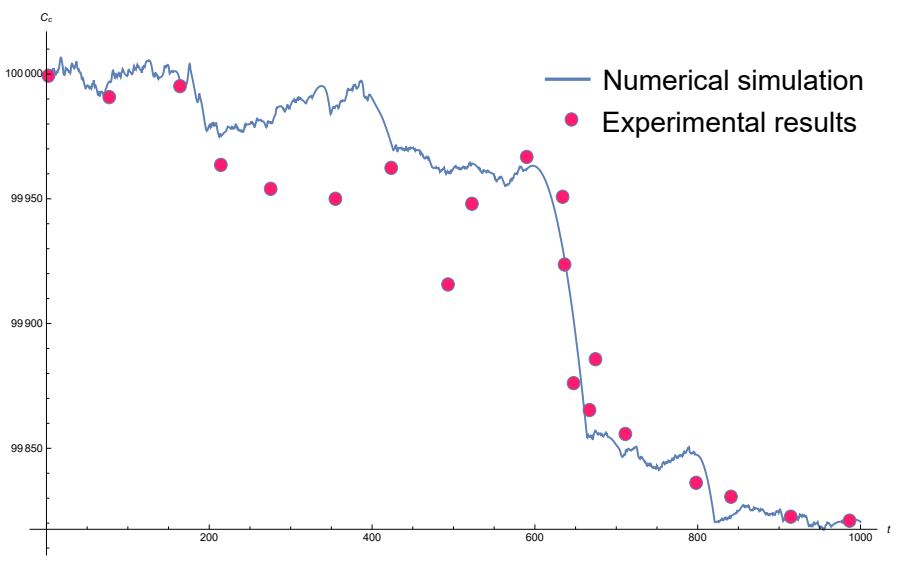

Figure 3: The solution profile of the cancer cells vs time. Patient No. 3.

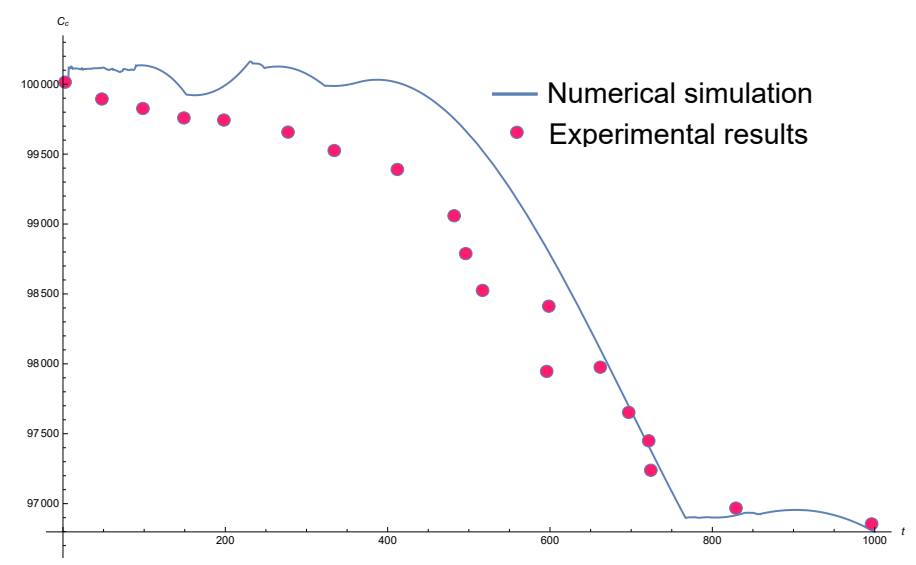

Figure 4: The solution profile of the cancer cells vs time. Patient No. 4.

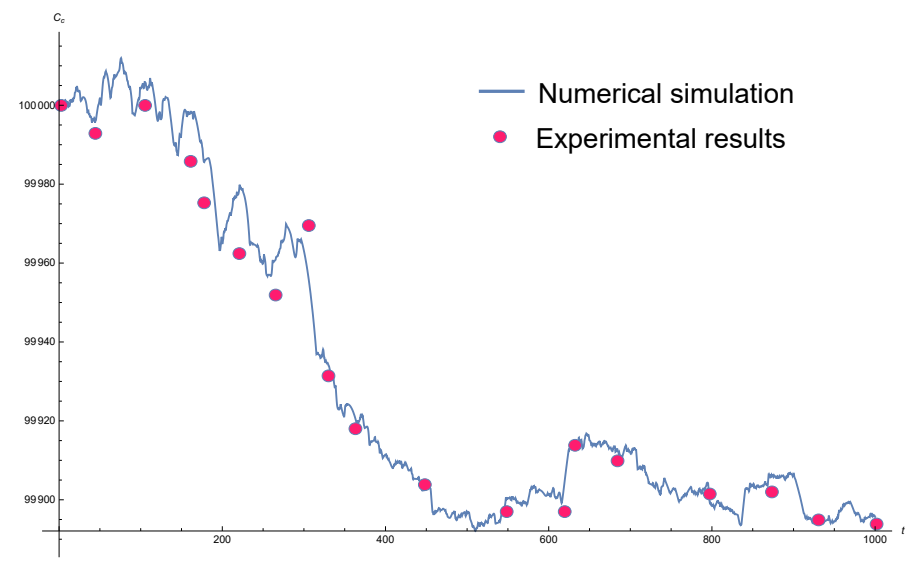

Figure 5: The solution profile of the cancer cells vs time. Patient No. 5. 


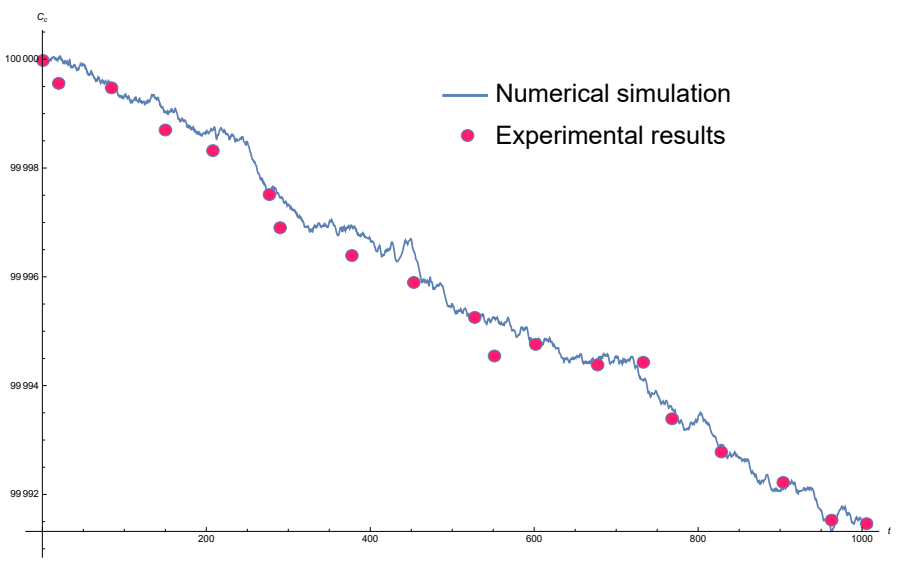

Figure 6: The solution profile of the cancer cells vs time. Patient No. 6 .

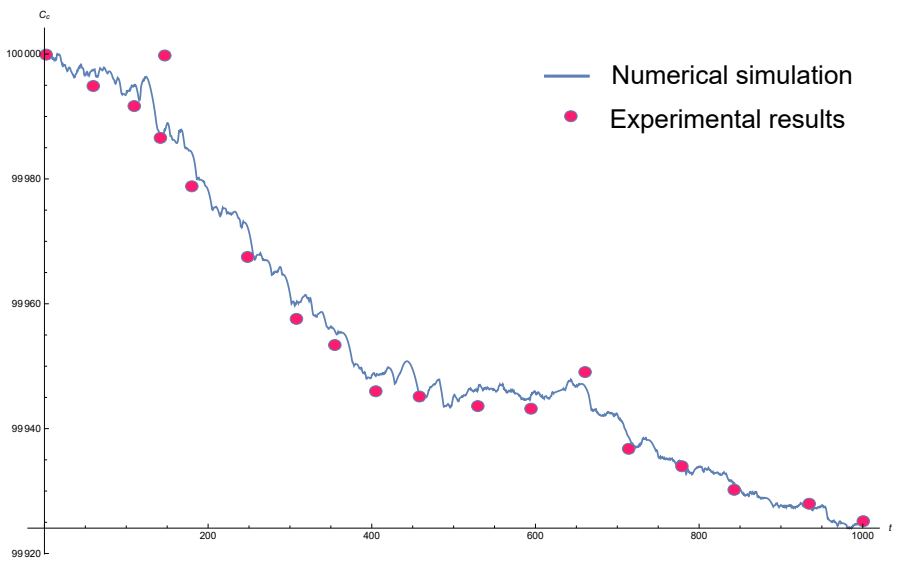

Figure 7: The solution profile of the cancer cells vs time. Patient No. 7. 


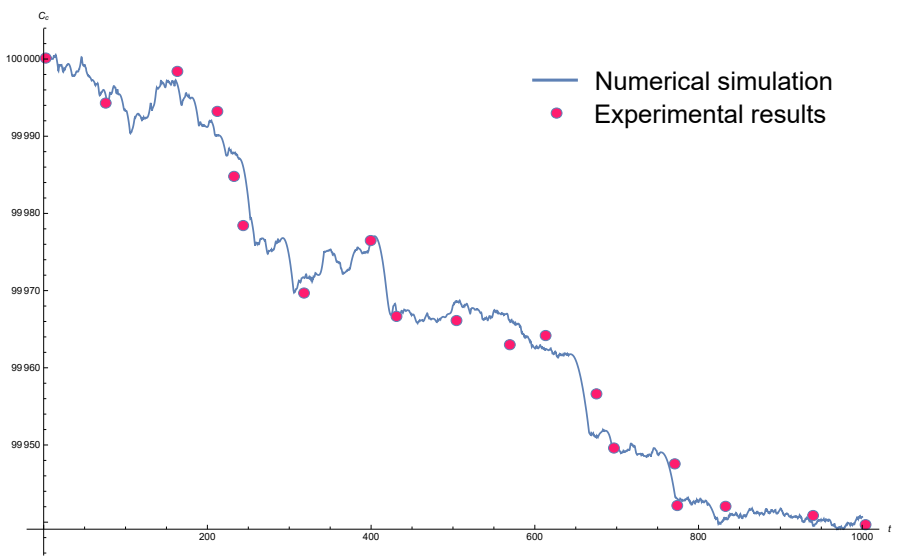

Figure 8: The solution profile of the cancer cells vs time. Patient No. 8.

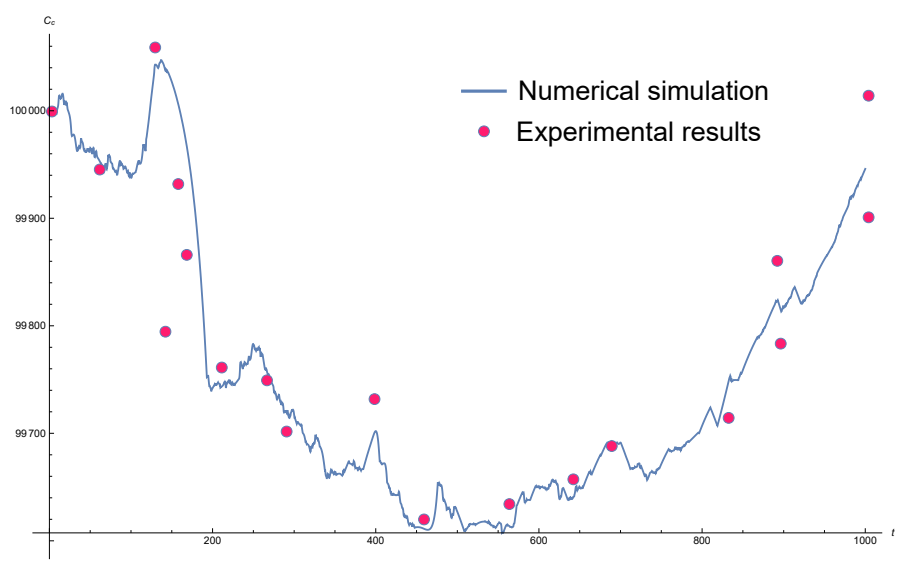

Figure 9: The solution profile of the cancer cells vs time. Patient No. 9. 


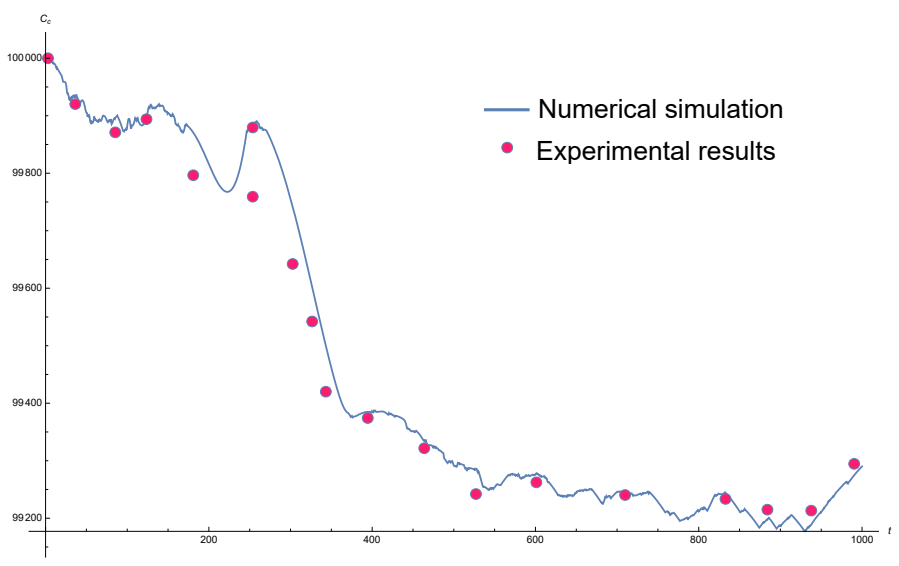

Figure 10: The solution profile of the cancer cells vs time. Patient No. 10.

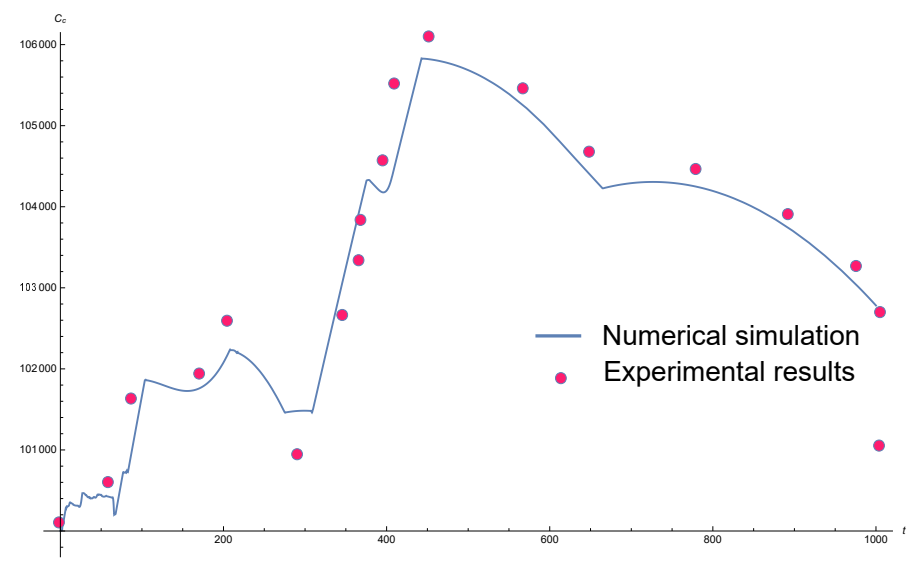

Figure 11: The solution profile of the cancer cells vs time. Patient No. 11. 


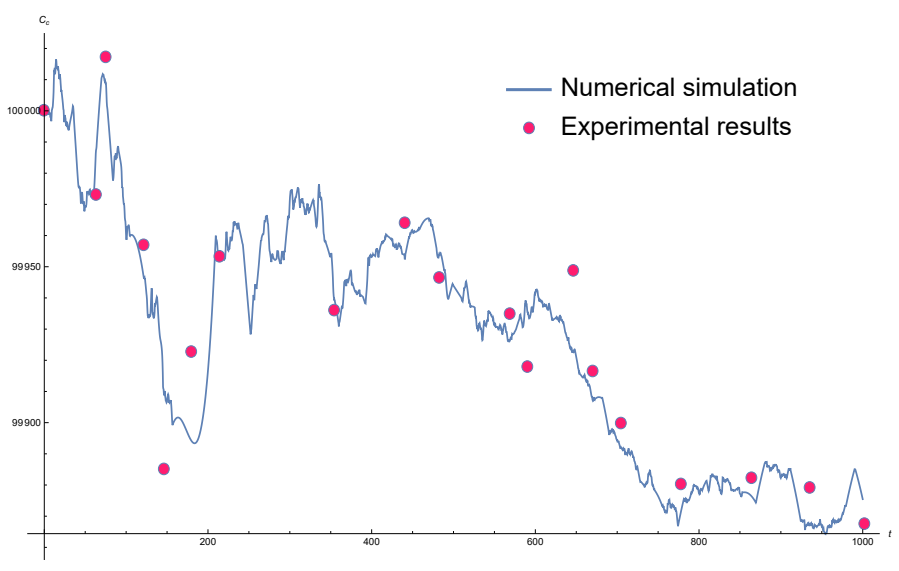

Figure 12: The solution profile of the cancer cells vs time. Patient No. 12.

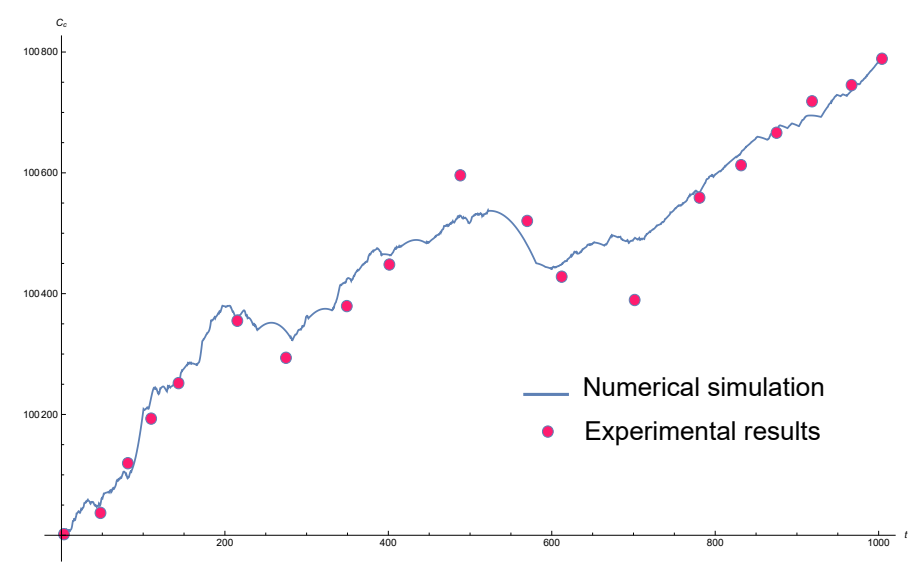

Figure 13: The solution profile of the cancer cells vs time. Patient No. 13. 


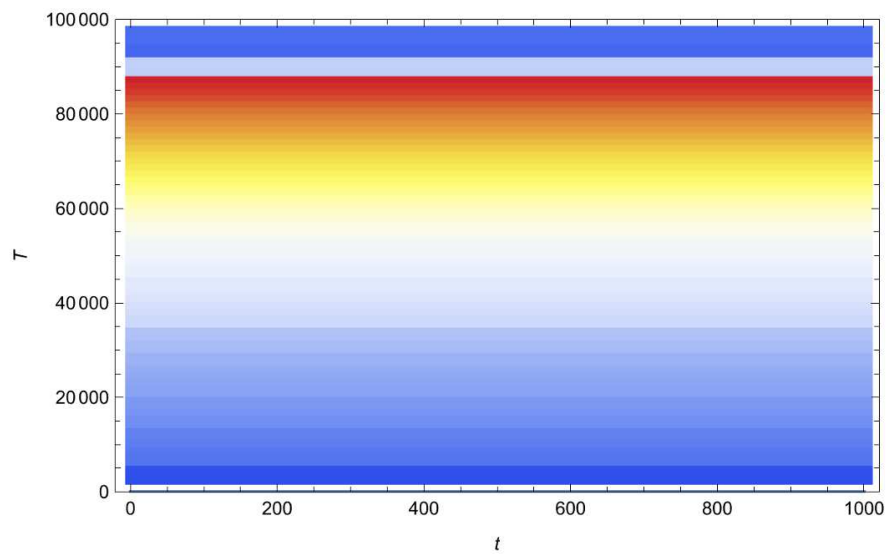

Figure 14: The density plot of the cancer cells vs time. Patient No. 1.

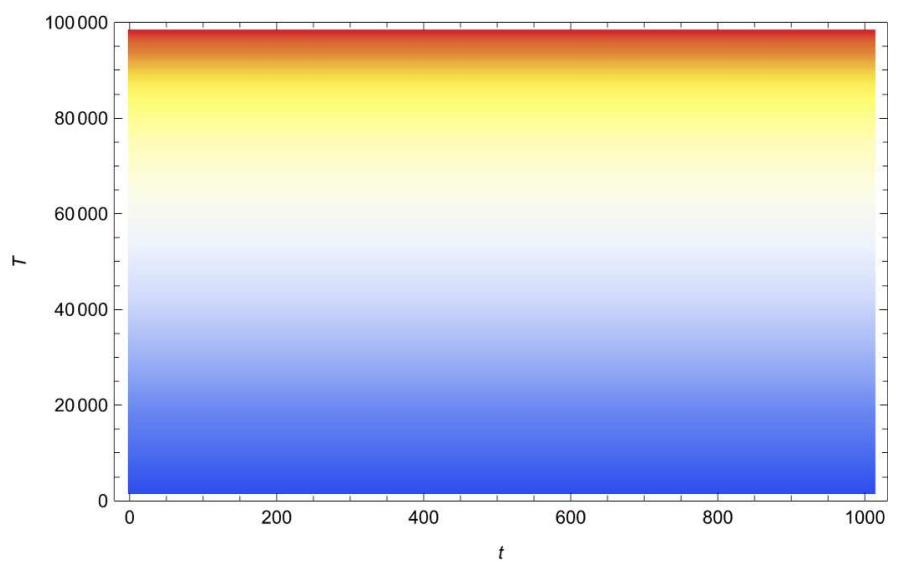

Figure 15: The density plot of the cancer cells vs time. Patient No. 2. 


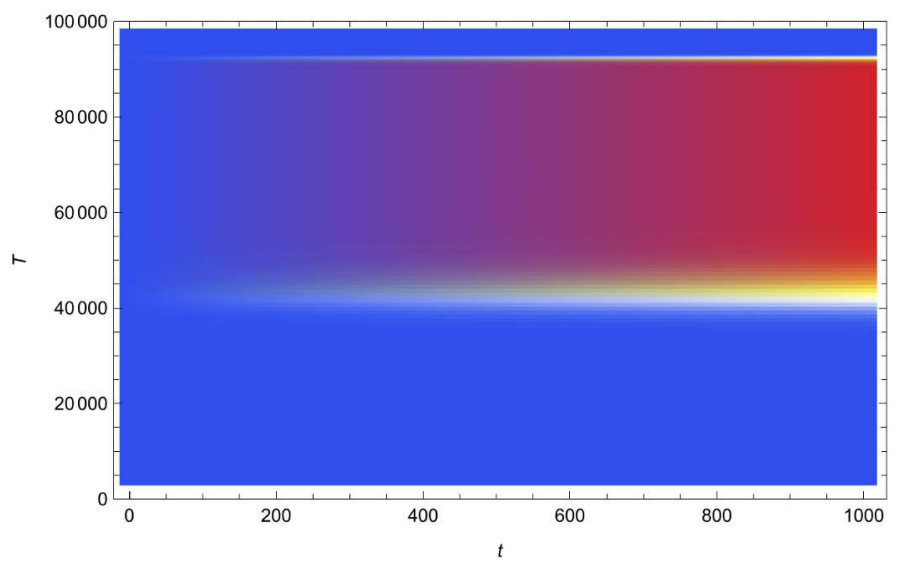

Figure 16: The density plot of the cancer cells vs time. Patient No. 3.

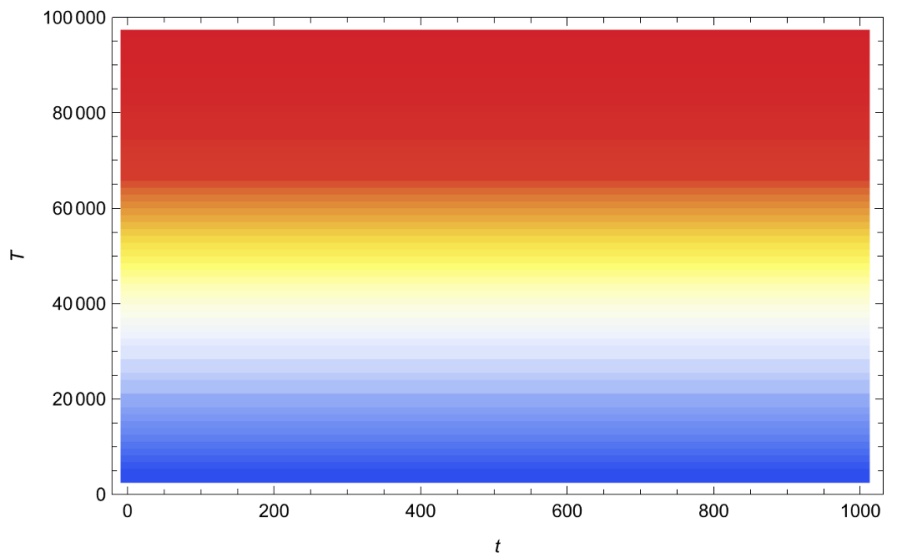

Figure 17: The density plot of the cancer cells vs time. Patient No. 4. 


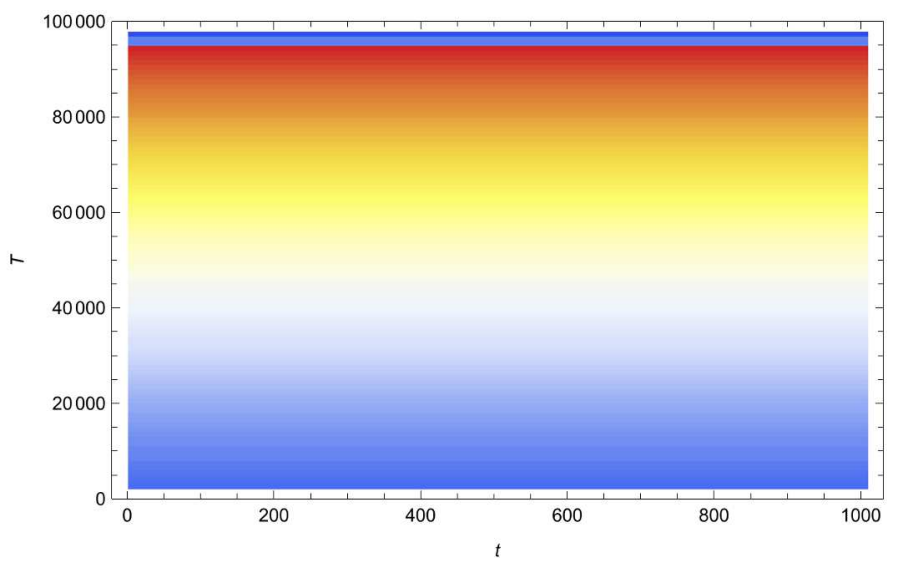

Figure 18: The density plot of the cancer cells vs time. Patient No. 5.

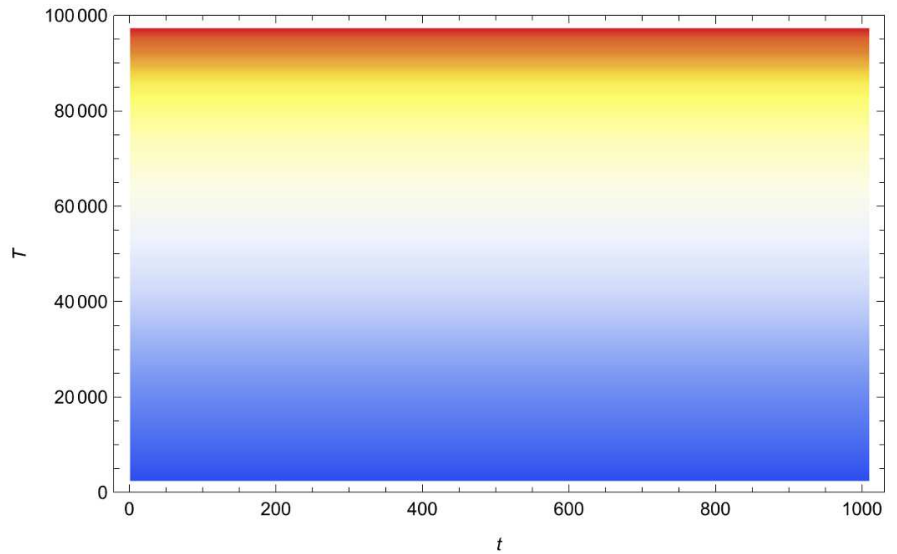

Figure 19: The density plot of the cancer cells vs time. Patient No. 6 . 


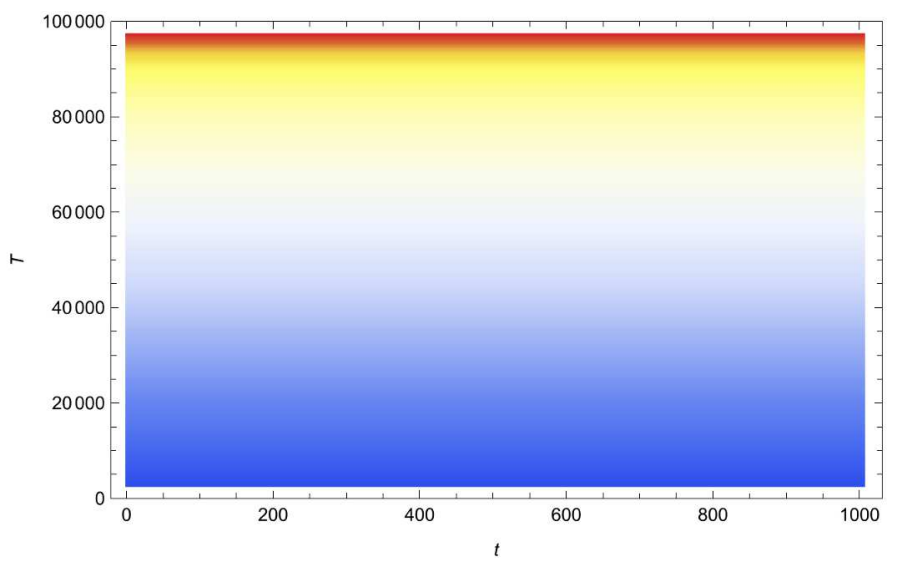

Figure 20: The density plot of the cancer cells vs time. Patient No. 7.

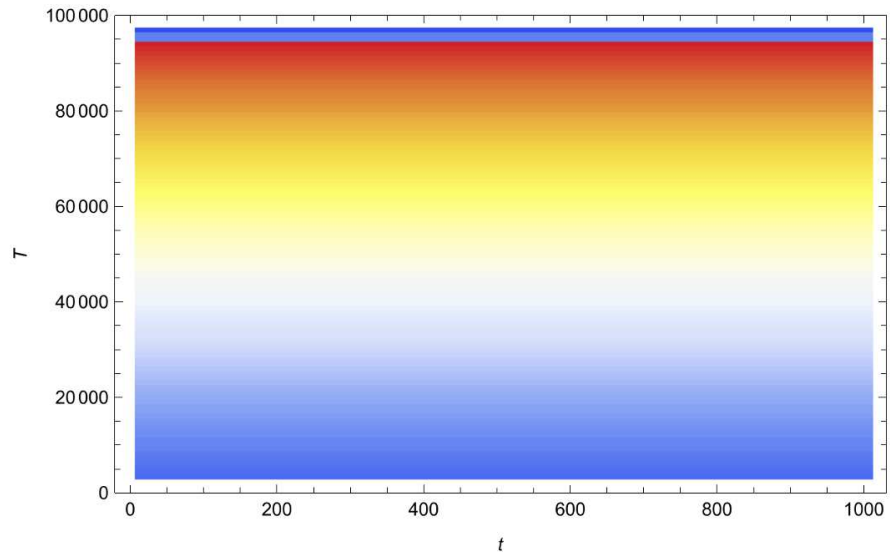

Figure 21: The density plot of the cancer cells vs time. Patient No. 8. 


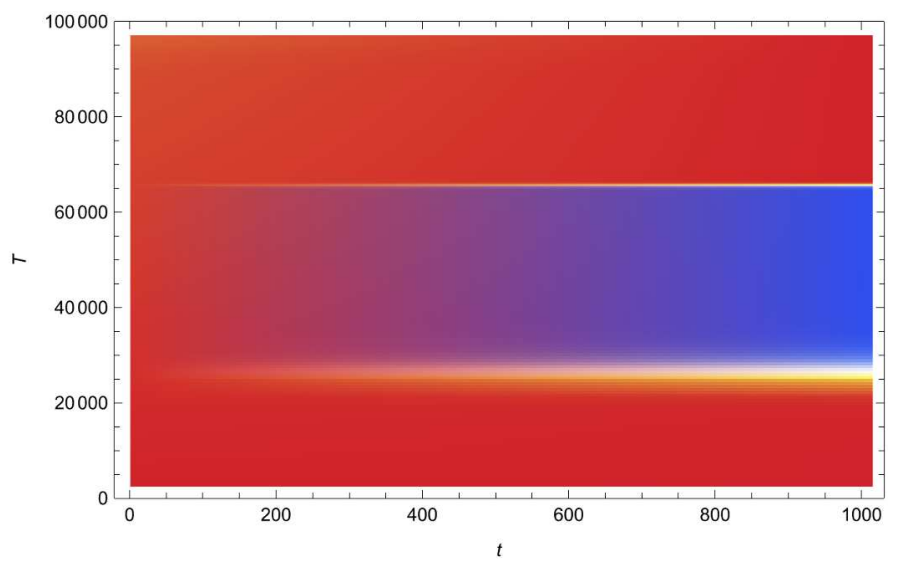

Figure 22: The density plot of the cancer cells vs time. Patient No. 9.

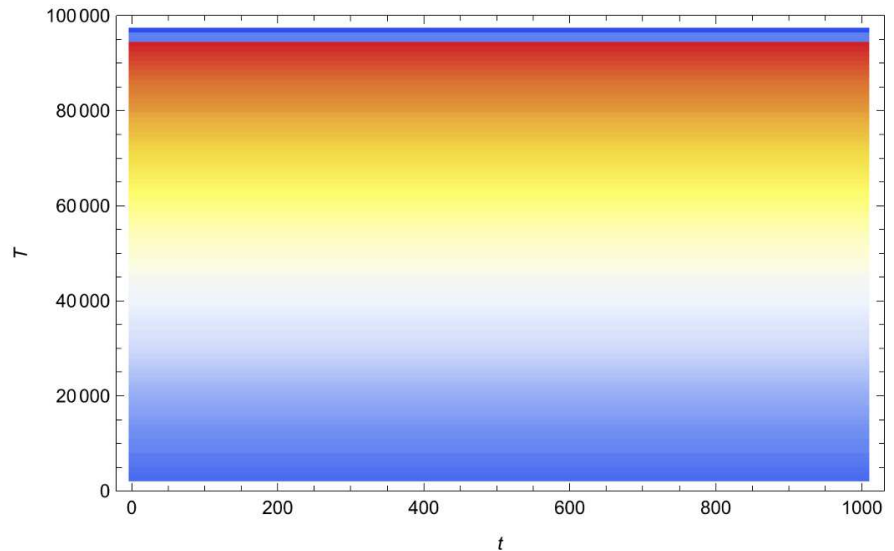

Figure 23: The density plot of the cancer cells vs time. Patient No. 10. 


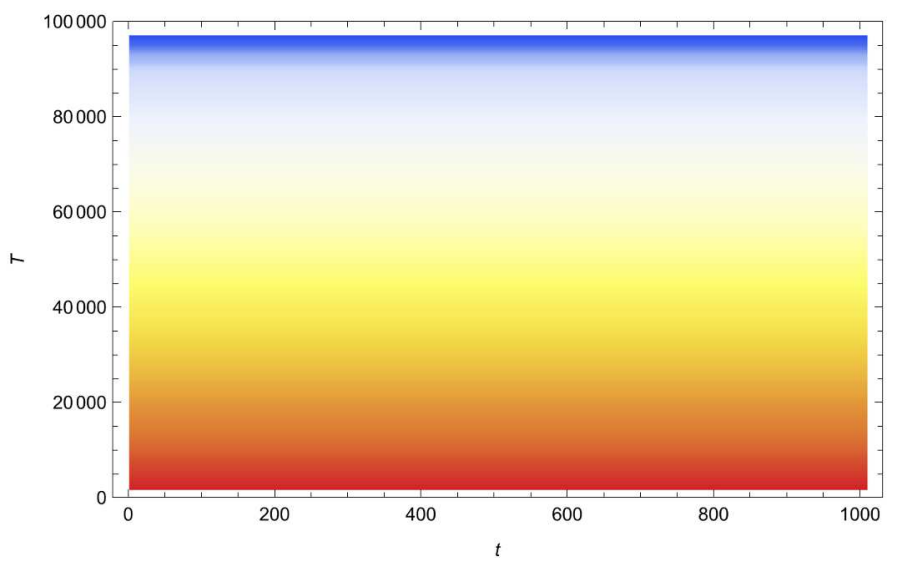

Figure 24: The density plot of the cancer cells vs time. Patient No. 11.

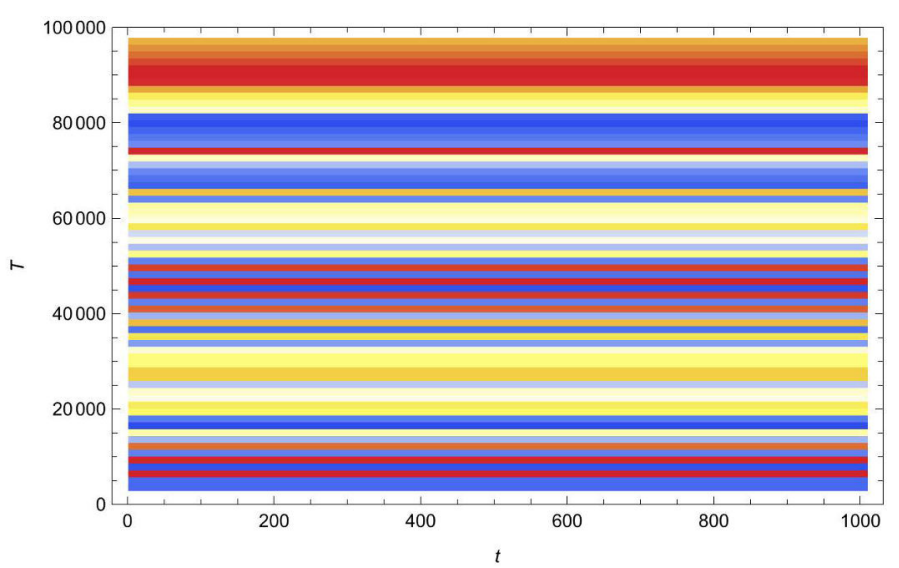

Figure 25: The density plot of the cancer cells vs time. Patient No. 12. 


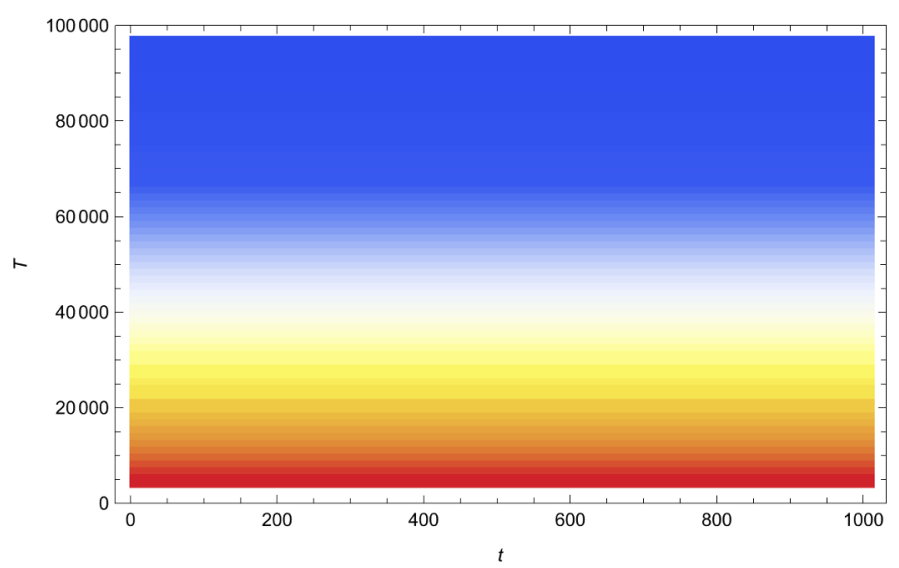

Figure 26: The density plot of the cancer cells vs time. Patient No. 13. 


\section{References}

[1] National Cancer Institute NIH: https://www.cancer.gov/aboutcancer/understanding/what-is-cancer.

[2] Cancer Research Institute: https://www.cancerresearch.org/

[3] Sandeep S., John P. S., Hermann B. F., Mauro F., John P. F. and Vittorio C. Mathematical modeling of cancer progression and response to chemotherapy, Expert Review of Anticancer Therapy, 6:1361-1376 (2006).

[4] Miguel A. M., Mathematical modeling of cancer metabolism, Critical Reviews in Oncology/Hematology, 124:37-40 (2018).

[5] Sahai N., Gogoi M. and Ahmad N., Mathematical Modeling and Simulations for Developing Nanoparticle-Based Cancer Drug Delivery Systems: A Review. Current Pathobiology Reports, 9:1â8 (2021).

[6] Vittorio C., and John L., Multiscale Modeling of Cancer An Integrated Experimental and Mathematical Modeling Approach, Cambridge University Press, (2010).

[7] M.R. Roussel and S.J. Fraser, Geometry of the steady-state approximation: perturbation and accelerated convergence methods, The Journal of Chemical Physics, 93:1072-1081 (1990)

[8] M.R. Roussel and S.J. Fraser, Invariant manifold methods for metabolic model reduction, Chaos, 196:196-206 (2001).

[9] A. Zagaris, H.G. Kaper, and T.J. Kaper, Analysis of the computational singular perturbation reduction method for chemical kinetics, Journal of Nonlinear Science, 14:59-91 (2004).

[10] Shijun L., Beyond Perturbation Introduction to the Homotopy Analysis Method, Chapman and Hall/CRC, (2003).

[11] Mark R. T., Ardith E. K. Alain G. A mathematical model of tumor-immune interactions, Journal of Theoretical Biology, 294:56-73 (2011).

[12] Luis S. O., Stacey D. F. A cancer treatment based on synergy between anti-angiogenic and immune cell therapies, Journal of Theoretical Biology, 394:197-211 (2016).

[13] Maas U., Pope S.B., Simplifying chemical kinetics: Intrinsic low-dimensional manifolds in composition space, Combustion and Flame, 88:239-264 (1992).

[14] Bongers H., Van Oijen J.A., De Goey L.P.H., Intrinsic low-dimensional manifold method extended with diffusion, Proceedings of the Combustion Institute, 29:1371-1378 (2002), 
[15] Bykov V., Goldfarb I., Gol'dshtein V., and Maas U., "On a modified version of ILDM approach: asymptotic analysis based on integral manifolds," in IMA Journal of Applied Mathematics, 71:359-382 (2006).

[16] Yu, C., Minuzzi, F., Bykov, V., Maas, U., Methane/Air Auto-Ignition Based on Global Quasi-Linearization (GQL) and Directed Relation Graph (DRG): Implementation and Comparison. Combustion Science and Technology, 192(9):1802â1824 (2019).

[17] Robertson-Tessi, Mark, et al. âA Mathematical Model of TumorâImmune Interactions.â Journal of Theoretical Biology, 294:56â73 (2012).

[18] Cameron M., Davis A. L., Kostelich E., Eikenberry S. A Mathematical Model of Angiogenesis in Glioblastoma Multiforme, Arizona State University (2009). Published 2009 